\title{
Correction to: Clinical characteristics and cardiovascular outcomes in patients with atrial fibrillation receiving rhythm-control therapy: the Fushimi AF Registry
}

\author{
Yoshimori An ${ }^{1}$ - Masahiro Esato ${ }^{2}$ Mitsuru Ishii ${ }^{1} \cdot$ Moritake Iguchi $^{1} \cdot$ Nobutoyo Masunaga $^{1} \cdot$ Hikari Tsuji $^{3}$. \\ Hiromichi Wada $\cdot$ Koji Hasegawa $^{4} \cdot$ Hisashi Ogawa ${ }^{1} \cdot$ Mitsuru Abe $^{1} \cdot$ Gregory Y. H. Lip ${ }^{5,6} \cdot$ Masaharu Akao $^{1}$
}

Published online: 27 June 2018

(c) Springer Japan KK, part of Springer Nature 2018

\section{Correction to: Heart and Vessels \\ https://doi.org/10.1007/s00380-018-1194-5}

In the original publication of the article, the data of Body mass index $\geq 25\left(\mathrm{~kg} / \mathrm{m}^{2}\right)^{\mathrm{a}}$ is published incorrectly in Table 1 . Since this is categorical variable, the data should have been expressed as a number (percentage), not as mean \pm standard deviation. The corrected version of Table 1 is as below:

The original article can be found online at https://doi.org/10.1007/ s00380-018-1194-5.

\section{Masaharu Akao}

akao@kuhp.kyoto-u.ac.jp

1 Department of Cardiology, National Hospital Organization Kyoto Medical Center, 1-1, Mukaihata-cho, Fukakusa, Fushimi-ku, Kyoto 612-8555, Japan

2 Department of Arrhythmia, Ijinkai Takeda General Hospital, Kyoto, Japan

3 Tsuji Clinic, Kyoto, Japan

4 Division of Translational Research, National Hospital Organization Kyoto Medical Center, Kyoto, Japan

5 Institute of Cardiovascular Sciences, University of Birmingham, Birmingham, UK

6 Aalborg Thrombosis Research Unit, Department of Clinical Medicine, Aalborg University, Aalborg, Denmark 
Table 1 Baseline characteristics and co-morbidities between the two groups in the entire cohort

\begin{tabular}{|c|c|c|c|}
\hline \multirow[t]{3}{*}{ Number } & \multicolumn{3}{|l|}{ Entire cohort } \\
\hline & Rhythm-control & Rate-control & $P$ value \\
\hline & 478 & 1279 & \\
\hline \multicolumn{4}{|l|}{ Baseline characteristics } \\
\hline Female $^{\mathrm{a}}$ & $172(36 \%)$ & $560(44 \%)$ & 0.003 \\
\hline Age $(\text { years })^{\mathrm{a}}$, mean $\pm \mathrm{SD}$ & $70.5 \pm 10.8$ & $74.3 \pm 10.4$ & $<0.001$ \\
\hline Age $\geq 75$ years & $183(38 \%)$ & $691(54 \%)$ & $<0.001$ \\
\hline Body mass index $\left(\mathrm{kg} / \mathrm{m}^{2}\right)$, mean $\pm \mathrm{SD}$ & $23.3 \pm 3.6$ & $23.0 \pm 4.1$ & 0.25 \\
\hline Body mass index $\geq 25\left(\mathrm{~kg} / \mathrm{m}^{2}\right)^{\mathrm{a}}$ & $104(28 \%)$ & $316(28 \%)$ & 0.96 \\
\hline Systolic blood pressure $(\mathrm{mmHg})$, mean $\pm \mathrm{SD}$ & $125.3 \pm 17.6$ & $121.7 \pm 18.4$ & $<0.001$ \\
\hline Diastolic blood pressure $(\mathrm{mmHg})$, mean $\pm \mathrm{SD}$ & $71.4 \pm 11.1$ & $69.2 \pm 13.1$ & 0.001 \\
\hline Pulse rate (beats $/ \mathrm{min})^{\mathrm{a}}$, mean $\pm \mathrm{SD}$ & $75.0 \pm 14.5$ & $78.9 \pm 16.3$ & $<0.001$ \\
\hline Paroxysmal $\mathrm{AF}^{\mathrm{a}}$ & $390(82 \%)$ & $428(33 \%)$ & $<0.001$ \\
\hline Previous cardiac device implantation & $40(8 \%)$ & $100(8 \%)$ & 0.71 \\
\hline Estimated GFR $\left(\mathrm{ml} / \mathrm{min} / \mathrm{m}^{2}\right)$, median, $(25 \%, 75 \%)$ & $50.5(40.8,61.1)$ & $48.5(36.8,60.0)$ & 0.17 \\
\hline \multicolumn{4}{|l|}{ Co-morbidities } \\
\hline $\mathrm{CHADS}_{2}$ score, mean $\pm \mathrm{SD}$ & $1.53 \pm 1.27$ & $2.21 \pm 1.28$ & $<0.001$ \\
\hline $\mathrm{CHA}_{2} \mathrm{DS}_{2}$-VASc score, mean $\pm \mathrm{SD}$ & $2.71 \pm 1.63$ & $3.64 \pm 1.62$ & $<0.001$ \\
\hline History of stroke/TIA ${ }^{\mathrm{a}}$ & $63(13 \%)$ & $229(18 \%)$ & 0.016 \\
\hline Heart failure $^{\mathrm{a}}$ & $48(10 \%)$ & $509(40 \%)$ & $<0.001$ \\
\hline Hypertension $^{\mathrm{a}}$ & $278(58 \%)$ & $812(63 \%)$ & 0.040 \\
\hline Diabetes mellitus ${ }^{\mathrm{a}}$ & $87(18 \%)$ & $306(24 \%)$ & 0.01 \\
\hline Coexistence of organic heart disease & $50(10 \%)$ & $238(19 \%)$ & $<0.001$ \\
\hline Valvular heart disease ${ }^{a}$ & $41(9 \%)$ & $326(25 \%)$ & $<0.001$ \\
\hline Coronary artery disease ${ }^{\mathrm{a}}$ & $50(10 \%)$ & $238(19 \%)$ & $<0.001$ \\
\hline Myocardial infarction ${ }^{\mathrm{a}}$ & $17(4 \%)$ & $106(8 \%)$ & $<0.001$ \\
\hline Cardiomyopathy $^{\mathrm{a}}$ & $6(1 \%)$ & $56(4 \%)$ & 0.002 \\
\hline Left ventricular hypertrophy ${ }^{a}$ & $5(1 \%)$ & $30(2 \%)$ & 0.064 \\
\hline Peripheral artery disease ${ }^{\mathrm{a}}$ & $14(3 \%)$ & $59(5 \%)$ & 0.10 \\
\hline Renal dysfunction ${ }^{\mathrm{a}}$ & $303(72 \%)$ & $915(75 \%)$ & 0.38 \\
\hline Chronic obstructive pulmonary disease $\mathrm{a}^{\mathrm{a}}$ & $20(4 \%)$ & $68(5 \%)$ & 0.33 \\
\hline Anemia $^{\mathrm{a}}$ & $42(10 \%)$ & $211(17 \%)$ & $<0.001$ \\
\hline History of major bleeding & $10(2 \%)$ & $49(4 \%)$ & 0.072 \\
\hline \multicolumn{4}{|l|}{ Prescription at baseline } \\
\hline Oral anticoagulant & $226(47 \%)$ & $858(67 \%)$ & $<0.001$ \\
\hline Warfarin & $188(39 \%)$ & $771(60 \%)$ & $<0.001$ \\
\hline Dabigatran & $25(5 \%)$ & $42(3 \%)$ & 0.058 \\
\hline Rivaroxaban & $12(3 \%)$ & $26(2 \%)$ & 0.54 \\
\hline Apixaban & $1(0.2 \%)$ & $19(1 \%)$ & 0.025 \\
\hline Anti-platelet drug & $111(23 \%)$ & $425(33 \%)$ & $<0.001$ \\
\hline Diuretics $^{\mathrm{a}}$ & $64(13 \%)$ & $546(43 \%)$ & $<0.001$ \\
\hline Loop diuretics & $37(8 \%)$ & $462(36 \%)$ & $<0.001$ \\
\hline Thiazide diuretics & $21(4 \%)$ & $49(4 \%)$ & 0.60 \\
\hline Spironolactone & $14(3 \%)$ & $222(17 \%)$ & $<0.001$ \\
\hline Renin-angiotensin system inhibitors ${ }^{\mathrm{a}}$ & $187(39 \%)$ & $652(51 \%)$ & $<0.001$ \\
\hline \multicolumn{4}{|l|}{ Echocardiography } \\
\hline $\mathrm{LV}$ ejection fraction $(\%)^{\mathrm{a}}$, mean $\pm \mathrm{SD}$ & $66.9 \pm 9.2$ & $60.4 \pm 13.0$ & $<0.001$ \\
\hline LV ejection fraction $\leq 50 \%$ & $6(2 \%)$ & $103(8 \%)$ & $<0.001$ \\
\hline LA dimension $(\mathrm{mm})^{\mathrm{a}}$, mean $\pm \mathrm{SD}$ & $40.2 \pm 6.0$ & $46.1 \pm 8.3$ & $<0.001$ \\
\hline
\end{tabular}

Categorical data are presented as number (\%). Continuous data are presented as mean \pm standard deviation (SD), or median and interquartile range $(25 \%, 75 \%)$ according to the distribution

$A F$ atrial fibrillation, TIA transient ischemic attack, renal dysfunction estimated glomerular filtration rate $<60 \mathrm{ml} / \mathrm{min} / \mathrm{m}^{2}$, anemia hemoglobin level $<11 \mathrm{~g} / \mathrm{dl}, L V$ left ventricular, $L A$ left atrium

${ }^{\text {a }}$ Potential independent variables relevant to the choice of rhythm- or rate-control therapy selected for logistic regression model to develop propensity score 